\title{
CoRoTmission highlights
}

\author{
Magali Deleuil ${ }^{1}$, Pascal Bordé ${ }^{2}$, Claire Moutou ${ }^{1}$ \\ and the CoRoT exoplanet science team \\ ${ }^{1}$ Laboratoire d'Astrophysique de Marseille, \\ 38 rue F. Joliot-Curie, 13388 Marseille Cedex 13, France \\ email: magali.deleuil@oamp.fr \\ email: claire.moutou@oamp.fr \\ ${ }^{2}$ Institut d'Astrophysique Spatiale, Bât. 121, 91405 Orsay, France \\ email: pascal.borde@ias.u-psud.fr
}

\begin{abstract}
The CoRoTspace telescope is detecting planets with the transit method for more than four years. Its tally includes hot jupiter planets with orbital periods up to 95 days but also the first Super-Earth, CoRoT-7b, whose density is similar to the Earth's one,as well as close-in brown dwarfs. We review the status of the CoRoT/Exoplanet program, including some elements of the multi step strategy of complementary observations. We then present some of the CoRoT exoplanetary systems and how they widen the range of properties of the close-in low mass population and contribute to our understanding of the properties of planets.
\end{abstract}

Keywords. planetary systems, techniques: photometric

\section{The CoRoT mission}

With the advent of space missions, the search of exoplanets has entered a new area. Launched in december 2006, CoRoT has opened the field, followed in march 2009 by Kepler. CoRoT is a modest size mission designed to explore the transiting exoplanet population at short orbital period. The second main goal of the mission is the study of stellar internal structure through asteroseismology. In addition, a number of fields in stellar physics benefit this extremely precise time-series photometry.

CoRoT flies on PROTEUS, a CNES recurrent plateform, and orbits the Earth on an inertial polar orbit at an altitude of $896 \mathrm{~km}$. CoRoT has two continuous viewing zones (CVZ) which are two almost circular regions of $10^{\circ}$ radius each, centered on the Galactic plane at right ascension $6^{h} 50^{m}$ and $18^{h} 50^{m}$ respectively. A small drift of the orbit has allowed to slightly extend these CVZ along the lifetime of the CoRoT mission. Observing any stellar field in one of these two directions minimizes the level of background light. These positions have been selected taking into account the stellar content of these regions, in terms of possible targets for both scientific programs. The observation strategy consists in observational periods ranging from $\simeq 25$ days up to nearly six months. In order not to be blinded by the Sun, every six months, in April and October, the satellite is rotated by $180 \mathrm{deg}$ with respect to the polar axis and fields in the opposite direction are pointed. These two CVZ allows to probe regions in the galactic plane with various stellar properties in terms of metallicity but also in terms of age, as thin and thick disk populations appear not mixed in exactly the same proportions in these low galactic fields (Gazzano et al. 2011). One might expect to get some insights into the physical conditions that prevail for planet formation mechanisms once a more complete planet statistics will be available.

Nominally scheduled for 3 years, the mission has been extended for three additional years, that is till March 2013. Up to September 2010, a total of 129326 light curves has been collected. Their duration ranges from 21 days up to 152 days for the longest 

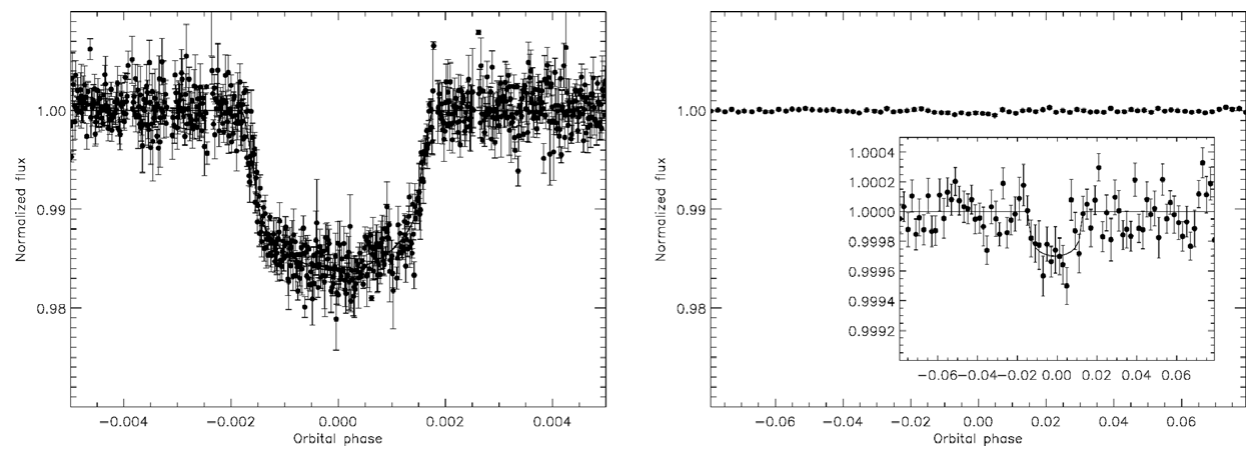

Figure 1. Two extreme cases of transit depth detected in the CoRoT light curves. Left : CoRoT- $9 b$ folded transit. Right : one of the smallest transit event plotted on the same scale, with a zoom on the transit.

run. The regular time sampling is $512 s$ which corresponds to the on-board co-addition of 16 exposures of $32 s$ each. This $32 s$ elementary integration time is preserved for one thousand of selected targets, referred to as over-sampled targets. During on-going CoRoT observations, the raw light curves are analyzed with the aim of detecting transits (Surace et al. 2008). According to this real-time detection called Alarm mode, the list of the over-sampled targets is updated, so that the possible forthcoming transit events will be accurately sampled at a cadence of $32 \mathrm{~s}$. In addition, this allows to start followup observations for the most promising candidates during the same observation season. Indeed, according to the mission policy, the CoRoT CoIs community accesses the data six months after the end of the observation of a given field, when the complete set of light curves is fully reduced and calibrated.

Transits are detected in about 100 up to 300 light curves per run. It gives a total of about 3000 light curves in which transits have been found so far. The depth of these transits ranges from $0.02 \%$ up to a few percents (Fig. 1). These transits are however predominantly eclipsing binaries. In that context, the long duration of the light curve is clearly an asset that allows to identify about $80 \%$ to $90 \%$ of the candidates as being stellar systems through secondary transits or light curve modulation. The remaining candidates are screened out by follow-up observations, using various techniques from photometric observations to radial velocity measurements. These follow-up observations play a major role in the CoRoT science and require a huge observational effort. They allow to assess the nature of the detected transiting body, stellar or planetary and, in the later case, to measure its mass. Bouchy et al. (2009) give a complete overview of the strategy for radial velocity observations and Deeg et al. (2009) present the ground-based photometric follow-up ones.

\section{Family portrait}

From 'Regular' hot Jupiters. Out of the 17 CoRoT planets secured at the time of the symposium, eleven belong to the giant planet population. As the regular members of this class, they exhibit a large spread in their orbital and physical characteristics. The planets density range from $\rho=7.3 \mathrm{~g} \mathrm{~cm}^{-3}$ for extremely dense CoRoT-14b (Tingley et al. 2011) to $\rho=0.22 \mathrm{~g} \mathrm{~cm}^{-3}$ in the case of CoRoT-5b whose host star is metaldepleted (Rauer et al. 2009). Three of them belong to the bloated population, that is planet whose radius is larger than can be accommodated by "standard" irradiated H-He 
planet models. There is still no clear consensus on the origin of these radius excesses. Tidally driven orbital evolution and the corresponding planet tidal heating even is one of the mechanisms regularly invoked (e.g. Jackson et al. 2008). This is the case of the very young CoRoT-2b system (Alonso et al. 2008) whose age is of a few Myr. Gillon et al. (2010) indeed reported a slight eccentricity of the orbit that might support such tidal origin.

Transit surveys being not affected by the same bias as the radial velocity method, it allows to enlarge the parameter space of host stars. Planet formation could be thus probed in new environments: around intermediate-mass stars that are often rapidly rotating or around metal depleted one. CoRoT-11b which orbits a fast rotating star with a $v \sin i=$ $40 \pm 5 \mathrm{~km} \mathrm{~s}^{-1}$, is one good example of this new class of planetary systems (Gandolfi et al. 2010).

Very few transiting planets present a marked eccentric orbit. With an eccentricity of $0.53 \pm 0.04$, CoRoT-10b is among the few exceptions. The planet whose orbital period is 13.2 days, experiences a 10.6-fold variation in insolation from the periastron to the apoastron Bonomo et al. (2010). At a much shorter orbital period of 5.35 days, CoRoT$16 b$, whose mass is $0.53 \pm 0.8 \mathrm{M}_{J u p}$ (Ollivier et al. 2011) is another possible case of eccentric orbit. The host star is faint with a $r^{\prime}$-mag of 15.5 and further radial velocity measurements are affected by large uncertainties. According to MCMC modeling, even though the circular orbit couldn't be completely rule out, an orbital solution with a value of $e=0.33 \pm 0.1$ is 30 times more probable. Kozai oscillations with a distant companion of stellar nature or by planet-planet scattering are the two mechanisms that might account for the high eccentricity. No hint of a non transiting companion from radial velocity monitoring or high angular resolution imaging has been so far reported for the two targets.

The long duration and high duty cycle of space-based observations allow to explore the transiting population over an extended range of orbital periods. Today CoRoT planets account for half of the transiting systems with an orbital period greater than 8 days. Among them, CoRoT-9b is of prime interest. First long orbital period transiting planet discovered by a transit survey, the planet orbits a solar like G3-type star in 95 days (Deeg et al. 2010). Its Jupiter's size and low eccentricity makes it a perfect representative of the largest known population of planets discovered by radial velocity surveys.
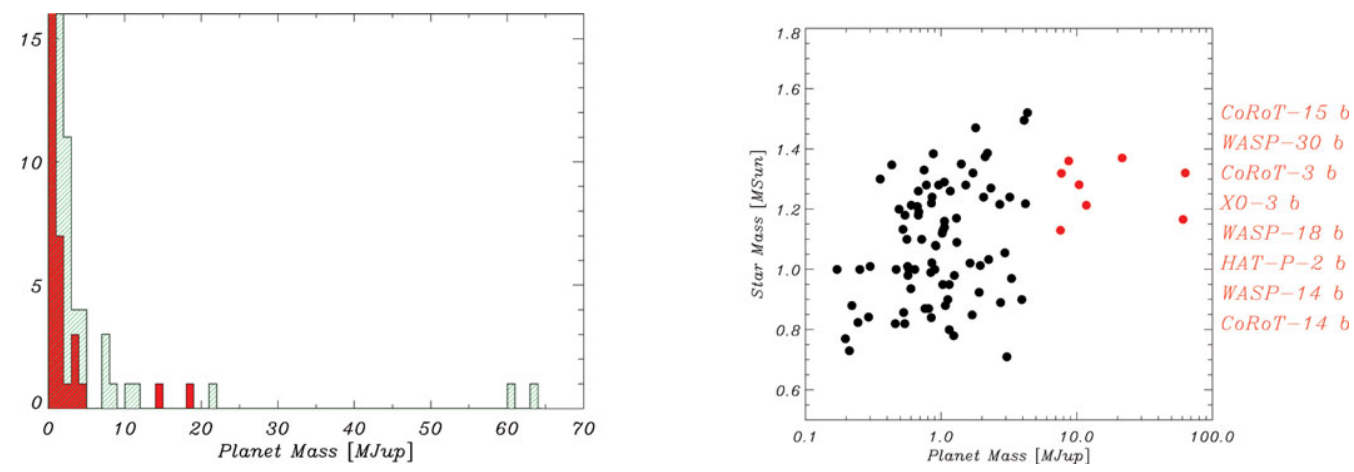

Figure 2. Left : Mass distribution of the exoplanets with orbital period less than 25 days; red: planets from radial velocity surveys, with a minimum mass only; green: transiting planets. Right : Mass of the host-star of transiting planets as a function of the mass of the planet. 
To super-massive. Up to 2008 , in mass range between 4 up to $\simeq 100 \mathrm{M}_{J u p}$, the mass distribution of the close-in population remained nearly un-populated. This paucity of massive sub-companions at short orbital period around solar-type stars was referred to as the brown-dwarf desert. As illustrated by the mass function distribution (Fig. 2), the transit surveys have recently started to populate this range of masses. First discovery of sub-stellar companion above the canonical value of $13 \mathrm{M}_{J u p}$ and an orbital period of about 4.5 days is CoRoT-3b (Deleuil et al. 2008). With a Jupiter's size but a mass of $21.6 \mathrm{M}_{J u p}$, it is located in the overlapping region between planets and brown dwarfs. According to planet formation models, core accretion or proto-planet collisions could result in planets with a mass up to $25 \mathrm{M}_{J u p}$. The exact nature of CoRoT-3b thus remains an open question as it could belong to a new population of massive planets or to the extreme tail of the brown dwarfs mass distribution. By contrast, its mass of $63.5 \mathrm{M}_{\text {Jup }}$ makes CoRoT$15 b$ a bonefide transiting brown dwarf (Bouchy et al. 2010). These two massive close-in companions transit a F-type star and so does the new transiting brown dwarf WASP-14b (A. Collier-Cameron et al. this conference). The host-star's mass plotted in Figure 2 as a function of the companion's mass shows that, up to date, all close-in companions with a mass greater than $10 \mathrm{M}_{J u p}$ are hosted by slightly more massive stars than the Sun, F-type star typically. These stars have usually a large $v \sin i$. The lack of detection by radial velocity surveys would be in that case a simple selection bias.
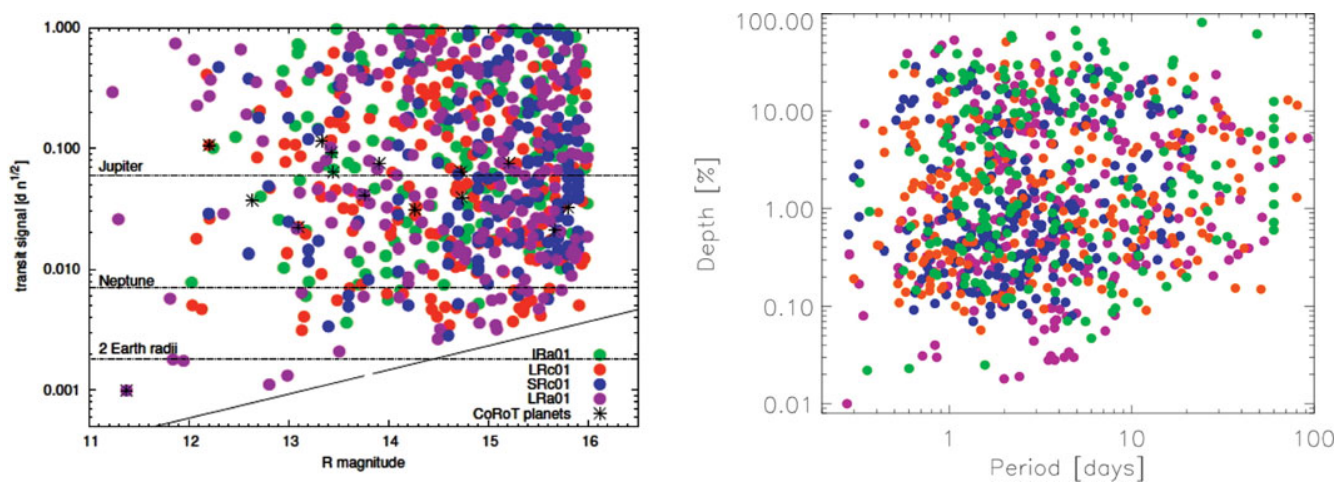

Figure 3. Left : Transit signal defined here as the product of the relative depth of the transit and the square root of the number of points in the transit, as a function of the CoRoT targets magnitude. Right: Depth of the transits as a function of the period of the event. The colored circles are all the candidates identified in the first runs according to the color codes in the bottom right of the left plot and the black stars the planets. (Adapted from Cabrera et al. (2009)).

And micro-planets. Space-based observations make the domain of small size planets around solar-like stars within reach. The detection capacity of CoRoT could be assessed using all the transit identified in the runs of the first year, whatever their nature, stellar or planetary, is. As illustrated in Figure 3, Neptune size planets can be detected whatever the magnitude of the star is, whereas super-Earth size planets down to 1 Earth radius can be discovered in the light curves of stars brighter than $\mathrm{R} \simeq 14$ only.

In the small-size regime, with a size of $0.57 \mathrm{R}_{J u p}$, CoRoT- $8 b$ has a density comparable to that of Neptune (Bordé et al. 2010), suggesting a massive core with a mass in the range 47 up to $64 \mathrm{M}_{\oplus}$ and a much smaller $\mathrm{H}$ - He envelope. In the mass - period diagram this planet lies in between the hot-Jupiter and the Super-Earth population, in a region where formation models predict a lack of planets. Its exact nature could be questioned as it might belong to the distribution tail of gaseous giants. Such massive planets with 
a high content of heavy elements are suggested to form preferentially around metal-rich stars, which is in agreement with the high metallicity of the host star.

With a depth of $0.35 \mathrm{mmag}$ CoRoT- $7 b$ is the smallest planet discovered so far orbiting a late K-type star. As illustrated by Léger et al. (2009), in this mass domain the main difficulty lies in establishing the exact nature of the transiting companion. Number of follow-up observations were required to rule out the various blend scenarii. Even more challenging is the estimate of the mass of the planet. The CoRoT-7a's stellar activity gives indeed a stellar jitter that dominates the radial velocity signal (Queloz et al. 2009) making the planet's mass subject to controversies. According to the different studies carried out on the same radial velocity measurement set, the estimated mass of the planet varies from $2 \mathrm{M}_{\oplus}$ (Pont et al. 2011) up to $6.9 \mathrm{M}_{\oplus}$ (Hatzes et al. 2010) with an uncertainty as large as $20 \%$. In addition, the exact number of planets in the system is also debated. There are indeed some evidences in the radial velocity signal for a second non-transiting planet (Queloz et al. 2009) and even a third one (Hatzes et al. 2010). But more data with an adapted observation strategy would be needed to clarify these issues.

\section{Probing the star - planet interactions}

Magnetic activity in the photosphere of the host stars could be also investigated thanks to the long temporal coverage of the photometric data. Various studies have mapped active regions on some of the CoRoT host stars (e.g. Lanza et al. (2011)). Among them, the young Sun-like star, CoRoT-2b has been intensively studied. Its light curve (Alonso et al. 2008) shows flux variations up to $6 \%$, related to the star's activity modulated by the stellar rotation. Different approaches have been used to reconstruct the surface active regions of the planet host star and follow their evolution. Using a maximum entropy spot modeling technique Lanza et al. (2009a) identified two active longitudes at the surface of the star, located on opposite hemispheres. This result is in good agreement with Huber et al. (2010) who used a different approach based on a modeling of the stars' surface over regular strips. Lanza et al. (2009a) found also evidence of a short-term spot cycle which suggests possible magnetic interaction between the star and its hot Jupiter-like planet.

Planet transits, acting like a magnifying glass, could also be used to probe spots physical characteristics. Modeling the series of 77 transits of CoRoT-2b across its parent star's stellar disk, Silva-Valio et al. (2010) estimated a spot's size ranging from 0.2 to 0.7 planet radius, the spots temperature being between 3600 and $5000 \mathrm{~K}$.

The stellar activity also impacts the planet's radius measurement by distorting the bottom of the transit. As a result, the transit depth is affected and could appear more shallower than in reality. In the case of CoRoT-2b, Silva-Valio et al. (2010) fitted the deepest transit of the series which yields a planet's radius $\simeq 3 \%$ larger than the value reported in the discovery paper which was calculated from the phase folded light curve.

Possible synchronization could also be searched for. The long duration light curves allows to derive the rotation period of the star. In the case of CoRoT-4b, Aigrain et al. (2008) established that it is a synchronized system, a result confirmed by Lanza et al. (2009b). Among the CoRoT planets, it remains however the only system for which the synchronization could be clearly detected.

\section{Summary}

At the time of the symposium, CoRoT has been in operation for 1383 days. With 17 planets fully characterized and a number of candidates still in the follow-up process, the instrument has fully demonstrated its ability to explore the close-in planet population 
over an extended range of sizes and properties from Super-Earth to temperate Jupitertype planets. Transit detection being not limited to solar-type stars, CoRoT has also unexpectedly started to populate the brown dwarf desert. Whatever their nature, massive planets or brown dwarf, the formation of these massive companions appears taking place around F-type stars.

High precision photometric observations over long time span have also proved being valuable to probe the stellar activity and search for new hints of star - planet interactions such as synchronization. It has also the potential to bring new interesting information on the stars activity. This being one of the main limitation in planet detection in the low mass domain that might help our understanding of the stellar signatures and further their modeling. With the mission extension and further improvements of the detection from a better understanding of the CoRoT light curves, new discoveries are expected. CoRoT will contribute further to get a better picture of the planetary systems characteristics.

\section{References}

Aigrain, S., Collier-Cameron, A., Ollivier, M., et al. 2008, A\&SA, 488, L43

Alonso, R., Auvergne, M., Baglin, A., et al. 2008, A\&A, 482, L21

Bonomo, A. S., Santerne, A., Alonso, R., et al. 2010, A\& $A, 520,65$

Bordé, P., Bouchy, F., Deleuil, M., et al. 2010, A\&A, 520, 97

Bouchy, F., Moutou, C., \& Queloz, D. 2009, Proc. IAU Symp. 253, 129

Bouchy, F., Deleuil, M., Guillot, T., et al. 2010, A\& A, 525, 68

Cabrera, J., Fridlund, M., Ollivier, M., et al. 2009, A\&A 506, 501

Deeg, H. J., Gillon, M., Shporer, A., et al. 2009, A\&A, 506, 343

Deeg, H. J., Moutou, C., Erikson, A., et al. 2010, Nature, 464, 384

Deleuil, M., Deeg, H. J., Alonso, R., et al. 2008, A $\mathscr{E} A$, 491, 889

Gandolfi, D., Hébrard, G., Alonso, R., et al. 2010, A\&A, 524, 55

Gazzano, J.-C., de Laverny, P., Deleuil, M., et al. 2011, (submitted to A\&GA)

Gillon, M., Lanotte, A. A., Barman, T., et al. 2010, A\&A, 511, 3

Hatzes, A. P., Dvorak, R., Wuchterl, G., et al. 2010, A\&A, 520, 93

Huber, K. F., Czesla, S., Wolter, U., \& Schmitt, J. H. M. M. 2010, A\&SA, 514, A39

Huber, K. F., Czesla, S., Wolter, U., \& Schmitt, J. H. M. M. 2009, A\& A, 508, 901

Jackson, B., Greenberg, R., \& Barnes, R. 2008, ApJ, 678, 1396

Lanza, A. F., et al. 2009, A\& $A, 493,193$

Lanza, A. F., et al. 2009, A\&A $A, 506,255$

Lanza, A. F., et al. 2011, A\&SA, 525, A14

Léger, A., et al. 2009, A\&\&A, 506, 287

Ollivier, M., et al. 2011, A\&A, to be submitted

Pont, F., Aigrain, S. \& Zucker S. 2011, MNRAS, 411, 1953

Queloz, D., Bouchy, F., Moutou, C., et al. 2009, A\&A, 506, 303

Rauer, H., Queloz, D., Csizmadia, Sz., et al. 2009, A\&A, 506, 281

Silva-Valio, A., Lanza, A. F., Alonso, R., \& Barge, P. 2010, textitA\&A , 510, A25

Surace, C., Alonso, R., Barge, P. et al. 2008, SPIE, 7019, 111

Tingley, B., Endl , M., Gazzano J.-C., et al., 2011, A\&A, 528, A97

Wolter, U., Schmitt, J. H. M. M., Huber, K. F., Czesla, S., Müller, H. M., Guenther, E. W., \& Hatzes, A. P. 2009, A\&A, 504, 561 